\title{
Evaluación de los efectos del deterioro sobre el potencial fisiológico de semillas de poroto (Phaseolus vulgaris L.) por prueba de tetrazolio
}

\author{
Effects of deterioration on the physiological potential of bean \\ (Phaseolus vulgaris L.) seeds evaluated by the tetrazolium test \\ Amalia Romano $^{1}$, Juan Argüello ${ }^{2}$, Irma Teves ${ }^{1}$, \\ Nora De Pascuale ${ }^{1}$, Gabriela Oddone ${ }^{3}$
}

\begin{abstract}
RESUMEN
Mediante pruebas de tetrazolio se evaluó la incidencia del deterioro sobre el potencial fisiológico de semillas de poroto. Para ello, muestras de Perla INTA (blanco) fueron sometidas a daño artificial, colocándolas en cámara de envejecimiento acelerado a $41{ }^{\circ} \mathrm{C}$ y $100 \%$ de humedad relativa, durante $0 ; 1 ; 2 ; 3$ y 4 días. Posteriormente cada sublote fue sumergido durante 25 minutos en una solución de tetrazolio al $0,5 \%$ a $40{ }^{\circ} \mathrm{C}$ en oscuridad. Como referencias para este análisis se evaluaron además el poder germinativo y la emergencia de plántulas de cada tratamiento. Para cada nivel de deterioro en estudió la proporción de semillas vigorosas estimadas por el test de tetrazolio con los resultados de germinación y emergencia de plántulas, no evidenció diferencias significativas $(\mathrm{p} \leq 0,05)$ entre ellos. Esto indica que el estudio con tetrazolio resulta adecuado para estimar diferencias de vigor entre lotes de semillas de poroto Perla INTA.
\end{abstract} semillas

Palabras clave: prueba de tetrazolio, deterioro de semillas de poroto (Phaseolus vulgaris L.), potencial fisiológico de

\begin{abstract}
Incidence of deterioration on the physiological potential of bean seeds has been analyzed by means of the tetrazolium test. With this purpose, samples of the Perla INTA (white) cultivar were submitted to artificial harm, by placing these in an accelerated aging chamber, at $41{ }^{\circ} \mathrm{C}$ and $100 \%$ RH, during 0; 1; 2; 3 and 4 days. Subsequently, each sublot was immersed during 25 minutes in a $0,5 \%$ tetrazolium solution at $40^{\circ} \mathrm{C}$, in the darkness. As references for this, germination power and seedling emergence of the treatment have been evaluated. For each level of deterioration being studied, the proportion of vigourous seed-estimated by the tetrazolium test-with the results of germination and seedlings emergence did'nt show significative differences $(p \leq 0,05)$ between them; thus indicating that the study with tetrazolium results adequate in order to estimate vigour differences between seed lots of Perla INTA bean.
\end{abstract}

Key words: tetrazolium test, deterioration of bean (Phaseolus vulgaris L.) seeds, physiological potential of seeds.

\section{Introducción}

Argentina aporta el $98 \%$ de la producción mundial de poroto alubia. El sostenimiento del mercado de este grano en la Unión Europea depende básicamente de la presentación de ofertas de mayor calidad. El área de este cultivo se localiza en los valles andinos de Salta, Jujuy, Catamarca, San Juan y Mendoza. Sin embargo, su limitada producción se debe al uso de semillas de baja calidad, problemática que demanda atención si se tiene en cuenta que la productividad de un cultivo depende entre otros factores de la calidad de la semilla que se utiliza (Carvalho et al., 2013). Además, el sector porotero enfrenta otras desventajas sostenidas por la carencia de conocimientos técnicos relacionados con la calidad de la semilla (SAGPYA, 2010), situación que le impide alcanzar un adecuado manejo agronómico de esta legumbre.

\footnotetext{
Universidad Nacional de Jujuy. Facultad de Ciencias Agrarias. San Salvador de Jujuy. Jujuy. Argentina.

Universidad Nacional de Córdoba. Facultad de Ciencias Agropecuarias. Córdoba. Argentina.

Universidad Nacional de Jujuy. Facultad de Ciencias Económicas. San Salvador de Jujuy. Jujuy. Argentina.

* Autor por correspondencia: amaliaromano@hotmail.com
}

Fecha de Recepción: 26 Diciembre, 2013.

Fecha de Aceptación: 31 Mayo, 2014. 
En general la semilla muestra su máximo potencial para la germinación y el vigor durante la madurez fisiológica. Posteriormente su calidad comienza a declinar debido a procesos naturales de deterioro (Krzyzanowski et al., 2008). Entre los factores que se reconocen determinantes del envejecimiento de la semilla se identifican aquellos de tipo ambiental (Trawatha et al., 1995) y otros de índole artificial inducidos durante la cosecha, en el almacenamiento y también durante la siembra (Tekrony, 2003). En ese sentido trabajos realizados en poroto sostienen que la expresión del deterioro varía en función del cultivar analizado (Romano, et al., 2008). Así, en esta línea de investigación se comprobó que poroto blanco es más sensible al deterioro artificial que poroto negro, al evidenciar mermas sostenidas en su calidad fisiológica según se incrementaba el daño (Romano, et al., 2010). Por otro lado, trabajando en poroto Santos et al. (2004) observaron que bajo condiciones de deterioro se producen cambios en sus sistemas enzimáticos. Así, utilizando la prueba de tetrazolio en semillas envejecidas de mijo (Basavarajappa et al., 1991) demostraron mermas graduales de la actividad deshidrogenasa según declinaba la calidad de la semilla.

Los test de vigor han sido ampliamente utilizados para identificar posibles diferencias en la calidad fisiológica entre lotes de semillas (Oliveira Diniz et al., 2013). Con ellos se evalúan características que determinan el potencial para una emergencia rápida y uniforme, así como el desempeño de plántulas normales en distintas condiciones ambientales (Schuab et al., 2006). Entre ellos se reconoce al test de tetrazolio que permite una rápida valoración tanto de la viabilidad como del vigor de la semilla (Cervi y Mendonca, 2009) y su eficiencia está relacionada con el desarrollo de una metodología adecuada para cada especie. Este ensayo se basa en alteraciones en la coloración de los tejidos seminales, en presencia de una solución de 2,3,5 cloruro de trifenil tetrazolio por la actividad de enzimas deshidrogenasas (Dias y Alves, 2008; Cervi y Mendonca, 2009). Así, mientras la sal de tetrazolio en semillas viables forma una sustancia insoluble llamada formazán, que se deposita en tejidos vivos y los colorea, en semillas no viables los tejidos muertos o deteriorados se presentan sin tinción. Estos resultados se probaron a partir de trabajos realizados en maní (Pérez y Argüello,
1995; Bittencourt y Vieira, 1996) y en soja (Vieira et al., 2010), quienes también observaron que según se altera el vigor de la semilla varía la intensidad de la tinción de los tejidos seminales de las especies analizadas, las que además demostraron su sensibilidad al daño.

Sobre la base de lo señalado, y considerando que en la actualidad no se dispone de patrones de tinción por tetrazolio que permitan evaluar la calidad de la semilla de poroto, se plantea como objetivo del presente trabajo elaborar un mapa de tinción para la determinación del vigor en poroto blanco cultivar Perla INTA.

\section{Materiales y métodos}

Material biológico: se utilizó un lote de semillas de poroto blanco (Phaseolus vulgaris L.) perteneciente al cultivar Perla INTA, cosecha 2010 de primera multiplicación, provista por la estación Experimental INTA-Cerrillos- Salta (Argentina).

Tratamiento para deterioro inducido: con el objeto de disponer de semillas con diferentes grados de daño, el lote de semillas de poroto previamente curado con captan a razón de $1 \mathrm{~g} / \mathrm{kg}$ y dividido en 4 sublotes, fueron llevados a cámara de envejecimiento acelerado a $41{ }^{\circ} \mathrm{C}$ y $100 \%$ de humedad relativa, durante: 0,1,2,3 y 4 días. Luego el material se dejó orear a temperatura de laboratorio hasta alcanzar la humedad inicial $(11,5 \%)$.

\section{Determinaciones en laboratorio}

Ensayo de germinación: se sembraron 4 repeticiones de 10 semillas entre toallas de papel humedecido con agua destilada. Los rollos ubicados en bolsas de polietileno transparente se colocaron en cámara de germinación con un fotoperiodo 8:16 horas (luz:oscuridad) a 20-30 ${ }^{\circ} \mathrm{C}$. Al noveno día se evaluó el número de plantas normales, anormales y el de semillas muertas (ISTA, 2010). Los resultados se expresaron en porcentajes.

Vigor por tetrazolio: este ensayo se repitió tres veces. Cuatro repeticiones de 50 semillas de poroto blanco de cada nivel de deterioro se embebieron entre toallas de papel húmedo con agua destilada por un periodo de 16 horas a $4{ }^{\circ} \mathrm{C}$. Posteriormente se retiró en forma manual el tegumento de cada semilla. Cada sublote fue sumergido en solución 
de tetrazolio al $0,5 \%$ a $40{ }^{\circ} \mathrm{C}$ en oscuridad durante 25 minutos, según determinaciones previas. A continuación las semillas fueron lavadas con agua corriente, y las muestras fueron evaluadas de acuerdo con la coloración adquirida por cada semilla y clasificadas en viables vigorosas; viables poco viables y no viables.

\section{Determinaciones a campo}

Emergencia de plántulas en sustrato de arena: este ensayo se llevó a cabo en San Salvador de Jujuy ( $\left.65^{\circ} 17^{\prime} \mathrm{W}, 24^{\circ} 11^{\prime} \mathrm{S}\right)$, Argentina, en el periodo 2010/2011. La arena utilizada fue previamente lavada, esterilizada y colocada en cajones de madera, procediéndose a sembrar 4 repeticiones de cada tratamiento. La humedad necesaria se mantuvo mediante aportes de riegos según demandas.

El conteo de las plántulas emergentes se realizó día por medio, a partir de la aparición de la primera plántula y durante 20 días. Los resultados se expresaron en porcentaje (Oliveira Diniz et al., 2013).

\section{Diseño experimental y análisis estadístico}

Para cada ensayo propuesto se utilizó un diseño completamente aleatorizado con 4 repeticiones. Con el uso de Excel, Infostat 2011 los datos fueron analizados por ANAVA, y las medias estudiadas por el test de Tukey $(p \leq 0,05)$.

\section{Resultados y Discusión}

A partir de los diferentes grados de deterioro en estudio -logrados mediante el envejecimiento acelerado- se observaron semillas de poroto blanco (Figura 1) con diferencias en la intensidad y localización de la coloración alcanzada por los tejidos seminales (Bittencourt y Vieira, 1996).

Los patrones comúnmente encontrados durante la tinción de la semilla son ilustrados y descritos en la Figura 1. Lo que pone de manifiesto la existencia de respuestas de la coloración que alcanzaron los tejidos según el nivel de daño que presentaba el lote analizado (Dias y Alves, 2008).

Las semillas viables vigorosas de poroto blanco se tiñen superficialmente de color rojo brillante y uniforme, y no muestran lesiones en el eje embrionario. Ello evidencia la presencia de tejidos vivos que mantienen actividad bioquímica y permeabilidad en sus membranas . Estos resultados concuerdan con apreciaciones realizadas en trigo por Carvalho et al. (2013), quienes recomiendan esta prueba para identificar y descartar lotes de semillas de inferior viabilidad.

Según avanza el envejecimiento de la semilla, declina su vigor, aparecen como consecuencia pérdidas en la integridad de las membranas (Schuab et al., 2006). Además entre otras alteraciones funcionales, se reconocen caídas en la actividad deshidrogenasa. Sobre el particular, en las semillas categorizadas como viables poco vigorosas y No vigorosas (Figura 1: C; D; E; F; G; H; I; J y K) los tejidos deteriorados mostraron áreas parciales de color rojo, rosado y/o sin tinción. Además al corte, los tejidos analizados evidenciaron escasa firmeza y localización de la coloración a mayores profundidades, debido a su mayor permeabilidad (Cervi y Mendonca, 2009).

Cuando se analiza el efecto del deterioro en semillas de poroto es necesario efectuar cuidadosas observaciones sobre las distintas porciones del embrión (Carvalho et al., 2013). Si los cotiledones se tiñen total o parcialmente (en áreas interiores o adyacentes a la superficie) de color rojo o rosado (Figura 1: A; B1 ; B2 y B3) son consideradas semillas vigorosas. Asimismo, los ejes embrionarios deben presentar un color rojo brillante y al corte el tejido debe ser firme (Bittencourt y Vieira, 1996).

Investigaciones realizadas en maní (Pérez \& Argüello, 1995) y en poroto (Romano et al., 2008) reconocen al eje embrionario como la porción de la semilla más sensible al daño. Esto se debe no solo por ser una parte vital para el crecimiento de una plántula normal, sino también porque deja semiexpuesta la radícula. Por lo tanto, a nivel de embrión solo daños superficiales podrán ser aceptados para categorizar a la semilla vigorosa. Es por lo señalado que en semillas viables poco vigorosas (Figura 1: C y D) se observan los extremos apicales de plúmula y en área central de cotiledones escasa tinción o nula tinción. Lo señalado se corresponde con apreciaciones realizadas en algodón por Cervi y Mendonca (2009). Estos autores verificaron concordancia entre las categorizaciones de semillas definidas por tetrazolio con los respectivos ensayos de germinación.

Por otra parte, cuando los tejidos seminales a nivel de radícula y/ó plúmula y los ubicados próximos al borde externo de los cotiledones (Figura 1: E; F y G) están muy deteriorados, las alteraciones funcionales evidencian declinaciones en la actividad 


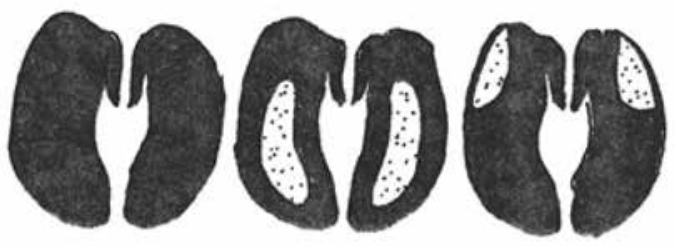

A

B 1

B 2

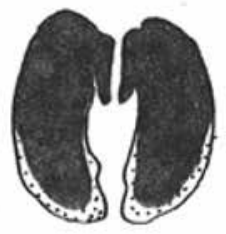

B 3

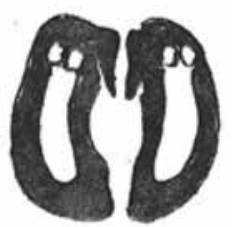

C

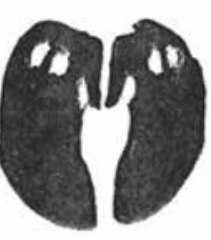

D

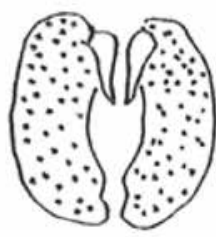

E

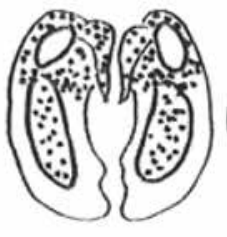

F

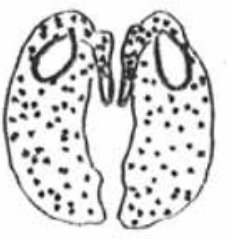

G

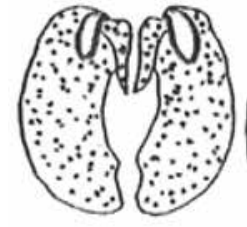

H

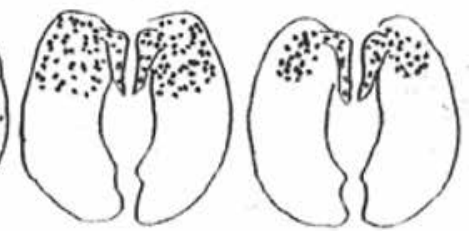

I

J

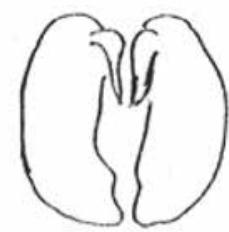

K

Figura 1. Patrón de tinción para la determinación del vigor por tetrazolio en semillas de poroto blanco cv. Perla INTA (áreas oscuras indican tinción rojo; áreas punteadas indican tinción rosada y áreas blancas muestran falta de coloración).

Detalles de la Figura 1

VIABLES VIGOROSAS: A. Eje embrionario completamente teñido de color rojo; B1; B2 y B3. Ejes embrionarios teñidos de rojo. Áreas interiores (central, superior e inferior) de cotiledones de color rosado; VIABLES POCO VIGOROSAS: C. Eje embrionario teñido de rojo. Coloración más clara en extremo de plúmula $(<1 \mathrm{~mm})$ y en el área central de cotiledones. D. Eje embrionario teñido de rojo, excepto extremo de plúmula $(<2 \mathrm{~mm})$. NO VIABLES: E. Eje embrionario teñido de rosado, excepto radícula completa. F. Eje embrionario teñido de rosado, excepto plúmula y contorno externo de cotiledones. G. Eje embrionario teñido de rosado, excepto plúmula y extremo de radícula. H. Eje embrionario teñido de rosado, excepto área de unión raíz-hipocotilo y porción del cotiledón. I. Eje embrionario con tinción clara, excepto más del 70\% de cotiledones sin colorear. J. Eje embrionario levemente teñido. Cotiledones sin coloración. K. Eje embrionario sin coloración. 
deshidrogenasa, por lo tanto se observan variaciones en la tinción (Dias y Alves, 2008), lo que permite inferir la calidad fisiológica de la semilla. Al respecto, en la categoría de semillas no viables la susceptibilidad al daño se expresa sin tinción en la unión raíz/hipocotilo (Figura 1: H); el eje embrionario con tenue coloración (Figura 1: I); cotiledones sin coloración (Figura 1: J) y el eje embrionario no teñido (Figura 1: K). Al respecto, Vieira et al. (2010) afirmaron que por su sensibilidad el test topográfico de tetrazolio puede ser utilizado para determinar diferencias en los niveles de deterioro en soja.

La Tabla 1 muestra para los distintos niveles de deterioro estudiados, los porcentajes de semillas viables vigorosas; viables poco vigorosas y no viables. Estas clases en forma aditiva se trasformaron en combinaciones, cuyos valores porcentuales se asociaron a la emergencia de plántulas (Tabla 2). Aquellas combinaciones que mostraron el valor de " $\mathrm{t}$ " más bajo integraron las respectivas categorías.
Por otra parte, los valores porcentuales totales de semillas viables vigorosas (Tabla 2) obtenidos de la combinación de la clases identificadas fueron comparadas con la emergencia a campo y el ensayo de germinación. El análisis estadístico realizado entre las mencionadas determinaciones no mostró diferencias significativas ( $\mathrm{p} \leq 0,05$ ), lo que permite verificar la concordancia entre la clasificación obtenida por la prueba de tetrazolio con los ensayos de germinación (Cervi y Mendonca, 2009), y con los obtenidos de la emergencia de plántulas (Shuab et al., 2006).

\section{Conclusiones}

Basado en los resultados obtenidos bajo las condiciones en las que se desarrolló el presente trabajo, se concluye que

- el avance en el deterioro de la semilla contribuye a la pérdida de su calidad fisiológica, lo que

Tabla 1. Categorías de tinción por tetrazolio de semillas de poroto blanco cv. Perla INTA, durante el envejecimiento acelerado.

\begin{tabular}{|c|c|c|c|c|c|c|c|}
\hline \multicolumn{3}{|c|}{ Categorías de tinción } & \multicolumn{5}{|c|}{ Porcentajes de semillas de cada tratamiento } \\
\hline Grupo & Descripción & Código & $\mathrm{T}$ & 1 & 2 & 3 & 4 \\
\hline \multirow[t]{2}{*}{$\begin{array}{l}\text { Viables } \\
\text { vigorosas }\end{array}$} & $\begin{array}{l}\text { Embrión totalmente teñido } \\
\text { de rojo. }\end{array}$ & A & 60 & 36 & 24 & 0 & 0 \\
\hline & $\begin{array}{l}\text { Embrión teñido de rojo, } \\
\text { coloración más clara en área } \\
\text { central superior y/o inf. de } \\
\text { cotiledón }\end{array}$ & B & 27 & 33 & 40 & 0 & 0 \\
\hline $\begin{array}{l}\text { Viables } \\
\text { poco } \\
\text { vigorosas }\end{array}$ & $\begin{array}{l}\text { Embrión teñido de rojo, } \\
\text { coloración más clara en } \\
\text { extremos de plúmula }(<1 \mathrm{~mm}) \\
\text { y área central de cotiledón. } \\
\text { Embrión teñido excepto } \\
\text { extremos de plúmula }(<2 \mathrm{~mm}) \text {. }\end{array}$ & $\mathrm{C}$ & 10 & 13 & 8 & 1 & 0 \\
\hline \multirow[t]{7}{*}{$\begin{array}{l}\text { No } \\
\text { viables }\end{array}$} & $\begin{array}{l}\text { Embrión teñido excepto } \\
\text { radícula. }\end{array}$ & $\mathrm{E}$ & 0 & 0 & 5 & 18 & 14 \\
\hline & $\begin{array}{l}\text { Embrión teñido excepto } \\
\text { plúmula y contorno externo } \\
\text { de cotiledones. }\end{array}$ & $\mathrm{F}$ & 2 & 4 & 5 & 20 & 25 \\
\hline & $\begin{array}{l}\text { Embrión teñido excepto } \\
\text { plúmula y extremo de radícula. }\end{array}$ & G & 0 & 2 & 4 & 21 & 30 \\
\hline & $\begin{array}{l}\text { Embrión teñido excepto } \\
\text { unión raíz/hipocotilo y zona } \\
\text { de cotiledones. }\end{array}$ & $\mathrm{H}$ & 1 & 2 & 3 & 11 & 0 \\
\hline & $\begin{array}{l}\text { Embrión con tinción clara, } \\
\text { excepto más del } 70 \% \text { de } \\
\text { cotiledones. }\end{array}$ & I & 0 & 4 & 2 & 14 & 9 \\
\hline & $\begin{array}{l}\text { Embrión levemente teñido. } \\
\text { Cotiledones sin coloración. }\end{array}$ & $\mathrm{J}$ & 0 & 3 & 2 & 8 & 10 \\
\hline & Embrión sin coloración. & K & 0 & 0 & 2 & 5 & 10 \\
\hline
\end{tabular}


Tabla 2. Ensayos de germinación, tetrazolio y emergencia de plántulas de poroto blanco cv Perla INTA durante el envejecimiento acelerado.

\begin{tabular}{|c|c|c|c|c|c|c|}
\hline \multirow{2}{*}{$\begin{array}{l}\text { Envejecimiento } \\
\text { acelerado (días) }\end{array}$} & \multicolumn{3}{|c|}{$\begin{array}{l}\text { Ensayos de germinación } \\
\qquad(\%)\end{array}$} & \multirow{2}{*}{$\begin{array}{c}\text { Tetrazolio } \\
(\%)\end{array}$} & \multicolumn{2}{|c|}{$\begin{array}{c}\text { Emergencia de plántulas } \\
(\%)\end{array}$} \\
\hline & Normales & Anormales & Muertas & & Agosto & Marzo \\
\hline Testigo & $89 a$ & 11 & 0 & $87 a$ & $78 \mathrm{a}$ & $66 a$ \\
\hline 1 & $69 \mathrm{~b}$ & 16 & 15 & $69 b$ & $75 a$ & $67 a$ \\
\hline 2 & $54 \mathrm{c}$ & 25 & 21 & $64 \mathrm{~b}$ & $49 b$ & $31 \mathrm{~b}$ \\
\hline 3 & Od & 0 & 100 & $0 \mathrm{c}$ & $1 \mathrm{c}$ & $2 \mathrm{c}$ \\
\hline 4 & Od & 0 & 100 & $0 \mathrm{c}$ & $0 \mathrm{c}$ & $0 \mathrm{c}$ \\
\hline
\end{tabular}

Cada valor representa la media de 4 repeticiones.

Letras diferentes indican diferencias significativas $(\mathrm{p} \leq 0,05)$ Tukey.

ha de influir en el stand final de plantas en el campo, como una consecuencia de las mermas en la germinación y el vigor de la semilla.

- la prueba de tetrazolio ha demostrado ser un sensible predictor de la calidad fisiológica de la semilla de poroto cv. Perla INTA.
- utilizando el mapa de tinción propuesto para poroto blanco, un técnico calificado en semillas podría estimar en forma rápida y segura la emergencia a campo de semillas de poroto del cultivar Perla INTA.

\section{Literatura Citada}

Basavarajappa, B.; Shetty, H. and Prakash, H.

1991. Membrane deterioration and other biochemical changes, associated with accelerated ageing of maize seeds. Seed Sci \& Technology, 19 (2): 279-286.

Bittencourt, S.; R. Vieira

1996. Use of reduced concentrations of tetrazolium solutions for the evaluation of the viability of peanut seed lots. Seed Sci and Technology, 25:75-82.

Carvalho,T.; F. Krzyzanowski; O. Castro Ohlson; M. Panobianco 2013. Tetrazolium test adjuntment for wheat seeds . Journal of Seed Sciencie, 35(3): 361-367.

Cervi, F.; E. Mendonca

2009. Adequacao do teste de tetrazolio para sementes de algodoeiro. Revista Brasileira de Sementes, 31(1): 177-186.

Colete, J.; R. Vieira; A. Dutra

2004. Electrical conductivity and soybean seedling emergence. Sci Agric.(Piracicaba.Braz), 61(4):386-391.

Dias, M.; S. Alves

2008. Avaliacao da viabilidade de sementes de Panicum maximum Jacq pelo teste de tetrazolio. Revista Brasileira de Sementes, 30(3): 152-158.

International Rules for Seed Testing

2010. Published by the International Seed Testing Association. Zürichstr.50.CH-8303. Bassersdorf, Switzerland.

Krzyzanowski, F.; J. Franca-Neto; A. Henning; N. Costa 2008. O controle de qualidade agregando valor a semente de soja. Serie Sementes.Londrina.Embrapa Soja. 12 p. (Embrapa Soja.Circular Técnica 54).

Oliveira Diniz, F.; M. Silva Reis; L. Santos Dias; E. Fontes Araujo; T. Sediyama; C. Atsumi Sedijama

2013. Physiological quality of soybean seeds of cultivars submitted to harvesting delay and its association witer seedling emergence in the field. Journal of Seed Science, 35(2): 147-152

Pérez, A.; J. Argüello

1995. Deterioration in peanut (Arachis hypogaea L. cv. Florman) seed under natural and accelerated aging. Seed Sci \& Technology, 23: 439-445.

Romano,A.; I. Teves; N. Torres; L. Cazón

2008.Variaciones en la calidad de semillas de poroto (Phaseolus vulgaris $\mathrm{L}$.) por efectos el daño mecánico y su influencia en el vigor de las plántulas.Idesia 26(2):83-87.

Romano, A.; I. Teves; L. Cazón

2010. Incidencia del daño mecánico en semillas de poroto (Phaseolus vulgaris L.) sobre el desarrollo y el rendimiento de plantas normales y anormales. Idesia 28(2):67-74.

Santos, C.M.R.; Menezes, N.L.; Villela, F.A.

2004. Alteracoes fisiológicas e bioquímicas em sementes de feijao envelhecidas artificialmente. Revista Brasileira de Sementes, 26 (1): 110-119.

Schuab, S.; A. de Lucca e Braccini, J. de Barros Franco Neto; C. Scarpini; D. Meschede

2006. Potencial fisiológico de sementes de soja e sua relacao com a emergencia das plántulas em campo. Acta Sci.Agron, 28 (4): 553-561.

Tekrony, D.

2003. Precision is and essential component in seed vigour testing.Seed Sci and Technology, 31: 435-447.

Trawatha, S.; D. Tekrony; D. Hilbrand 1995. Soybean lipoxigenase mutants and seed longevity. Crop Science, 35: 862-868.

Vieira, B.; R Vieira; F. Krzizanowski; J. Neto

2010. Alternative procedure for the cold test for soybean seeds. Sci Agric. (Piracicaba, Braz.), 67 (5): 540-545. 